\title{
African-American Men with Low-Risk Prostate Cancer: Modern Treatment and Outcome Trends
}

\author{
Augustine C. Obirieze - Ambria Moten • Delenya Allen • \\ Chiledum A. Ahaghotu
}

Received: 6 August 2014 / Revised: 27 September 2014 / Accepted: 24 October 2014 / Published online: 16 December 2014

(C) W. Montague Cobb-NMA Health Institute 2014

\begin{abstract}
Objective To investigate the clinical characteristics and treatment patterns for African-American (AA) men with low-risk prostate cancer (PCa) using a national, population-based dataset.

Methods We conducted a retrospective review of the Surveillance Epidemiology and End Results database 2004-2008. AA men aged $\geq 40$ years with low-risk PCa were identified. For comparison, white men were selected using the same selection criteria. We reviewed all recorded treatment modalities. Definitive treatment (DT) was defined as undergoing radiotherapy or prostatectomy.

Results Overall, 7246 AA men and 47,154 white men met the criteria. Most of the patients had PSA level between 4.1 and $6.9 \mathrm{ng} / \mathrm{mL}(56.2 \%)$ and received DT (76\%). Black men were younger (mean age: $62( \pm 8)$ vs. $65( \pm 10)$ years), less likely to receive DT (adjusted odds ratio (AOR), 0.71 [0.67-0.76]), and of those receiving DT, less likely to undergo prostatectomy (AOR, 0.58 [0.54-0.62]). Patients receiving DT had lower
\end{abstract}

This study was presented at the American Urological Association 2012 Annual Meeting, May 19-23, 2012, Atlanta, Georgia.

A. C. Obirieze · A. Moten • C. A. Ahaghotu

Outcomes Research Center, Department of Surgery, Howard

University College of Medicine, Washington, DC, USA

D. Allen - C. A. Ahaghotu

Department of Surgery, Howard University Hospital, Washington, DC, USA

\section{A. Ahaghotu}

Division of Urology, Department of Surgery, Howard University College of Medicine and Howard University Hospital, Washington, DC, USA

\section{A. Ahaghotu $(\bowtie)$}

Howard University Hospital, 2041 Georgia Ave, NW, Room 4C02, Washington, DC 20060, USA

e-mail: cahaghotu@howard.edu crude cancer-specific and overall mortality ( 0.17 vs. $0.41 \%$ and 2.9 vs. $7.8 \%, p$ value $<0.001$, respectively, among blacks). The difference in overall mortality was largest among $\geq 75$ years (5.6 vs. $18.2 \%$ ). Across age groups, blacks had higher all-cause mortality (AOR, 1.45 [1.13-1.87] and $1.56[1.31-1.86]$ for $<65$ and $\geq 65$ years, respectively).

Conclusion Our study of a large modern cohort of men with low-risk PCa demonstrates significant lower receipt of DT, lower receipt of prostatectomy among those receiving DT, and lower survival for black men compared to their white counterparts. Older men were less likely to receive DT. Patients who received DT had better survival. The survival difference was most striking among the elderly.

Keywords African-American · Low-risk prostrate cancer · Definitive treatment · Outcomes

\section{Introduction}

Prostate cancer is the most frequently diagnosed malignancy and second most common cause of cancer death among men in the USA [1]. According to the American Cancer Society, an estimated 241,740 new cases and 28,170 deaths are anticipated for the year 2012 [1].

Both the incidence and mortality for prostate cancer remain much greater among African-American men compared to other racial/ethnic groups, with incidence rates almost twice as high that of Caucasians, and mortality rates more than twice that of Caucasians [1]. Also, African-American men have been consistently shown to be diagnosed with more advanced disease at presentation. Several reasons have been given for the disparity gap [2-5].

With the advent of the prostate-specific antigen (PSA) era, coupled with technological advances in diagnosis of prostate cancer, there has been an increase in the proportion of prostate 
cancer diagnosis being made early in the course of the disease [6].

In addition to technical advances, the advent of the prostate-specific antigen (PSA) has led to the diagnosis and treatment of patients with earlier stages of prostate cancer and potentially higher cure rates [7]. While local therapy is associated with excellent long-term survival among patients with clinically localized disease, all available treatment exerts a potential morbidity and adverse effects on quality of life [6].

The treatment for localized prostate cancer remains controversial due to a lack of conclusive studies comparing the various forms of radiation to surgery. In the USA, radical prostatectomy and external beam radiation have been the most common forms of treatment. In $1995,34.1 \%$ of all patients with clinically localized disease underwent radical prostatectomy, and $26.3 \%$ were treated with external beam radiation. A mere $2.2 \%$ of the patients underwent brachytherapy [2]. Technological advances in the 1980s led to a resurgence of interest on radioactive seed implantation. The development of ultrasound guidance and improved computerized algorithms for determining optimal seed configuration lessened morbidity and allowed uniform dose homogeneity to become achievable. Permanent prostrate brachytherapy has now emerged as a mainstream treatment option for clinically localized prostrate cancer.

The continuing disparity of prostate cancer in the African-American population requires a closer examination. Therefore, we identified, using a population-based database, a cohort of African-American men with lowrisk prostate cancer with the goal of investigating demographic and clinical characteristics, as well as treatment patterns.

\section{Materials and Methods}

\section{Data Source}

The Surveillance Epidemiology and End Results (SEER) Program, maintained by the National Cancer Institute, is a public-use tumor registry that collects information on incidence, treatments and survival from populationbased cancer registries in the USA. Currently, SEER registries cover approximately $28 \%$ of the US population. Its incidence and mortality rates have been observed to be generally representative of the total US population [8]. Data collected by the registries include demographics, pathological/clinical, initial treatment, follow-up, and vital status. The methods of data collection as well as the structure of the SEER program have been described elsewhere [9].
Study Population

Patients' record with a diagnosis of prostate cancer in the SEER program 1973-2008 were identified using SEER code 28010, which corresponds to International Classification of Disease for Oncology, Third Edition (ICD-O-3) code of C619. Because of our interest in African-American men with low-risk disease, we selected patients identified as black non-Hispanic who met criteria for low-risk disease. Patients younger than 40 years or missing data on age information were excluded. Information on PSA, which is required for definition of low-risk disease, only became available in the SEER database from 2004, making us limit the study period to 2004-2008. For the purpose of comparison, non-Hispanic white patients satisfying the above criteria were also identified and included in the study. The final study cohort included 54,400 patient records (Fig. 1).

\section{Definitions}

As has been done in previous studies [10,11], low-risk disease was defined, using American Joint Committee on Cancer clinical stage, prostrate-specific antigen (PSA) level, and Gleason score, as stage T2a or lower, a PSA level of $\leq 10 \mathrm{ng} / \mathrm{mL}$, and Gleason score of 6 or less. Information on PSA values and Gleason score became available in the SEER program public-use dataset beginning in 2004, and the recorded PSA value is the highest laboratory value recorded in the medical record prior to diagnostic biopsy or treatment. PSA values were categorized as $\leq 2.5,2.6-4,4.1-6.9$, or 7 $10 \mathrm{ng} / \mathrm{mL}$.

The recorded Gleason score was based on addition of the primary and secondary patterns of the Gleason grade. Records

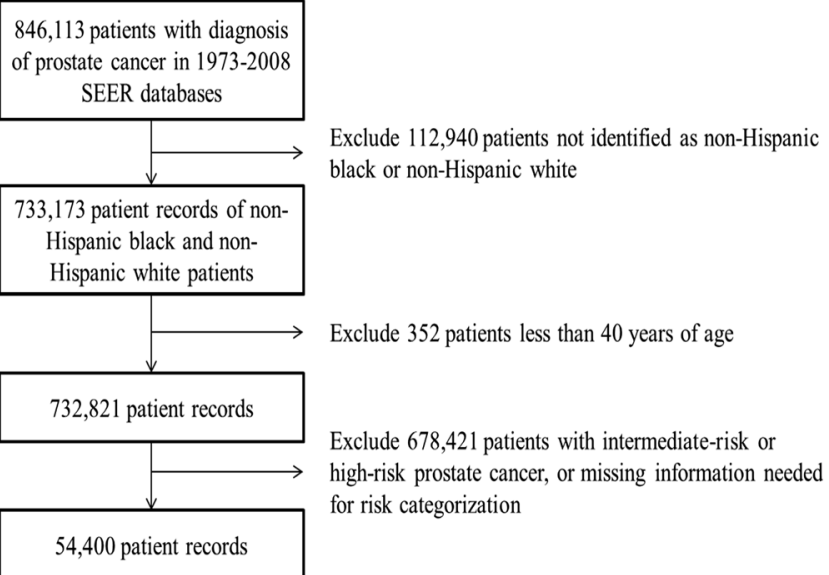

Fig. 1 Selection of study population. SEER surveillance epidemiology and end results 
having only one pattern on the Gleason grade, in which the number is less than five, are coded as missing, whereas those with such single pattern number greater than or equal to five had the number assumed to be a score. Gleason scores were classified as 2-3, 4-5, or 6 .

Available data on treatment type include radiation therapy and surgery. Radiotherapy was categorized into beam radiation, radioactive implants/radioisotope, or combination of beam radiation and radioactive implants/radioisotopes. Surgery included local excision, radical prostatectomy with/without pelvic lymphadenectomy. For the purpose of this study, definitive treatment (DT) was defined as receiving radiotherapy, undergoing surgery, or a combination of radiotherapy and surgery.

Table 1 Demographic and clinical characteristics of study population, $n=54,400$

\begin{tabular}{|c|c|c|c|}
\hline & Black, $n=7246$ & White, $n=47,154$ & $p$ value \\
\hline Age (years), median (IQR) & $62(56-68)$ & $65(59-71)$ & $<0.001$ \\
\hline \multicolumn{3}{|l|}{ Age group (years), $n(\%)$} & \multirow[t]{5}{*}{$<0.001$} \\
\hline $40-54$ & $1335(18.4)$ & $5383(11.4)$ & \\
\hline $55-64$ & $2940(40.6)$ & $17,887(37.9)$ & \\
\hline $65-74$ & $2417(33.4)$ & $17,565(37.3)$ & \\
\hline 75 or older & $554(7.7)$ & $6319(13.4)$ & \\
\hline PSA level (ng/mL), median (IQR) & $5.4(4.3-6.9)$ & $5.2(4.2-6.7)$ & $<0.001$ \\
\hline \multicolumn{3}{|l|}{ PSA level (ng/mL), $n(\%)$} & \multirow[t]{5}{*}{$<0.001$} \\
\hline$\leq 2.5$ & $551(7.6)$ & $4501(9.6)$ & \\
\hline $2.6-4$ & $796(11.0)$ & $6020(12.8)$ & \\
\hline $4.1-6.9$ & $4153(57.3)$ & $26,419(56.0)$ & \\
\hline $7-10$ & $1746(24.1)$ & $10,214(21.7)$ & \\
\hline \multicolumn{3}{|l|}{ Gleason score, $n(\%)$} & \multirow[t]{4}{*}{0.327} \\
\hline $2-3$ & $36(0.5)$ & $184(0.4)$ & \\
\hline $4-5$ & $236(3.3)$ & $1467(3.1)$ & \\
\hline 6 & $6974(96.3)$ & $45,503(96.5)$ & \\
\hline Definitive treatment, $n(\%)$ & $5312(73.3)$ & $36,021(76.4)$ & $<0.001$ \\
\hline \multicolumn{3}{|l|}{ Definitive treatment modality ${ }^{\mathrm{a}}, n(\%)$} & \multirow[t]{4}{*}{$<0.001$} \\
\hline Radiotherapy & $3437(64.7)$ & $20,237(56.2)$ & \\
\hline Prostatectomy & $1829(34.4)$ & $15,529(43.1)$ & \\
\hline Prostatectomy plus radiotherapy & $46(0.9)$ & $255(0.7)$ & \\
\hline \multicolumn{4}{|l|}{ County-level SES proxy ${ }^{\mathrm{b}}$, mean (SD) } \\
\hline Median household income ${ }^{c}$ & $9.20(2.06)$ & $9.77(2.39)$ & $<0.001$ \\
\hline Unemployed, $\%$ & $4.3(1.1)$ & $3.8(1.1)$ & $<0.001$ \\
\hline White collar occupation, $\%$ & $34.9(6.5)$ & $35.5(6.9)$ & $<0.001$ \\
\hline Living below poverty, $\%$ & $13.9(5.5)$ & $11.6(5.4)$ & $<0.001$ \\
\hline Less than high school education, $\%$ & $8.4(4.3)$ & $7.9(4.7)$ & $<0.001$ \\
\hline Urban housing, $\%$ & $92.0(16.2)$ & $86.2(20.9)$ & $<0.001$ \\
\hline Crude all-cause mortality, $n(\%)$ & $303(4.2)$ & $1382(2.9)$ & $<0.001$ \\
\hline Crude cancer-specific mortality, $n(\%)$ & $17(0.23)$ & $108(0.23)$ & 0.926 \\
\hline \multicolumn{4}{|c|}{ Crude cancer-specific mortality by age and intervention, $\mathrm{n}(\%)$} \\
\hline \multicolumn{4}{|l|}{ Younger $(<65$ years $)$} \\
\hline Definitive treatment received & $4(0.12)$ & $13(0.07)$ & 0.294 \\
\hline Definitive treatment not received & $2(0.21)$ & $5(0.14)$ & 0.583 \\
\hline \multicolumn{4}{|l|}{ Older ( $\geq 65$ years $)$} \\
\hline Definitive treatment received & $5(0.25)$ & $40(0.24)$ & 0.933 \\
\hline Definitive treatment not received & $6(0.60)$ & $50(0.67)$ & 0.800 \\
\hline
\end{tabular}

$P S A$ prostate-specific antigen, $S E S$ socioeconomic status, IQR interquartile range

${ }^{a}$ Among those who received definitive treatment

${ }^{\mathrm{b}}$ Represent proxy measures of socioeconomic status for the county of residence

${ }^{\mathrm{c}}$ In US\$5000 
Given the unavailability of data on socioeconomic status (SES) in the SEER public-use database, the US Census data was utilized to obtain SES-proxy variables. These countylevel data were merged to the SEER database using unique Federal Information Processing Standards (FIPS) county codes, and include median household incomes, percentages of persons with less than high school education by age 25 years, unemployed, living in urban areas, living below poverty, and with white collar occupations, Since the study cohort comprised patients diagnosed between 2004 and 2008, the 2000 US Census data were used.

\section{Statistical Analyses}

Univariate and bivariate analyses were performed to provide descriptive statistics. On bivariate analyses, Pearson chi-squared test was used to test distribution of categorical variables; student $t$ test was used to test difference in means between two groups; and nonparametric Fischer's exact test of medians was used to compare medians. Multivariable logistic regression analyses compared black patients to their white counterparts on the likelihood of receiving DT (and of undergoing surgery), adjusting for age, SEER registry, time trend, socioeconomic factors, PSA level, and Gleason score. Further risk-adjusted analyses also compared black and white patients across age groups $(<65$ and $\geq 65$ years), with age, type of intervention (stratified as no DT, radiotherapy, prostatectomy, and prostatectomy plus radiation), SEER registry, socioeconomic factors, time period, PSA level, and Gleason score as covariates. The much lower cancer-specific mortality incidence rates precluded a similar multivariable analysis, and in place we conducted bivariate analyses stratified by age and whether or not DT was received. All statistical analyses were two sided and statistical significance was defined as $p<0.05$. STATA/MP version 11.0 (Statacorp, College Station, TX, USA) was used to perform statistical analyses.

\section{Results}

A total of 7246 black men and 47,154 white men aged 40 years or older met our study criteria. Overall, the mean $( \pm \mathrm{SD})$ age at diagnosis was $64( \pm 10)$ years, and $12.6 \%$ of patients were 75 years or older. Most of the patients had PSA level between 4.1 and $6.9 \mathrm{ng} / \mathrm{mL}(56.2 \%)$, Gleason score of 6 (96.5\%), and received DT (76\%), comprising radiation therapy alone $(57.3 \%)$, prostatectomy alone $(42.0 \%)$, and prostatectomy with radiotherapy $(0.7 \%)$. Most patients who had radiation therapy had radioactive seed implant $(47.5 \%$ ), while $7.1 \%$

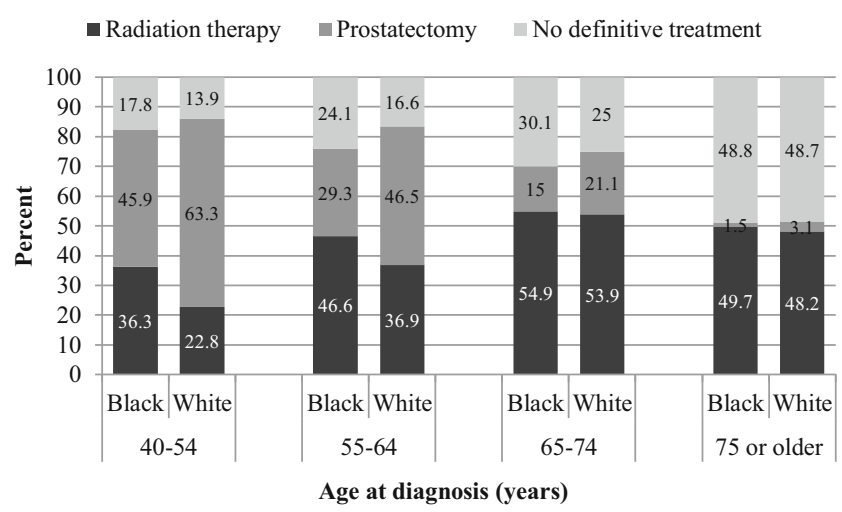

Fig. 2 Distribution of treatment type by age group and race

received a combination of external beam radiation and seed implant.

Table 1 shows the demographic and clinical characteristics of the black and white subcohorts. Black patients were younger (median age, 62 vs 65 years; $p<0.001$ ), had slightly higher PSA levels (median PSA level, 5.4 vs. $5.2 \mathrm{ng} / \mathrm{mL} ; p<0.001$ ), and among patients receiving DT, more likely to receive radiotherapy ( $64.7 \%$ vs. $56.2 \%, p<0.001)$ compared to white patients. Across all age groups of patients, black patients were less likely to undergo surgical treatment ( 45.9 vs. $63.3 \%, 23.3$ vs. $46.5 \%, 15$ vs. $21.1 \%$, and 1.5 vs. $3.1 \%$ for patients 40 $54,55-64,65-74$, and $\geq 75$ years, respectively) compared to their white counterparts (Fig. 2), and among those who received radiation therapy, black patients across all age groups were less likely to receive radioactive seed implant and more likely to receive beam radiation (Fig. 3). Black patients who did not receive DT were more likely to be aged 75 years or older compared to those who received DT (13.9 vs. $5.4 \% p$ value $<0.001)$. Among black patients, patients who received DT had lower crude all-cause mortality (2.9 vs. $7.8 \%$, $p<0.001)$ and crude cancer-specific mortality (0.17 vs. $0.41 \%, p<0.001)$ compared to patients who did not receive DT, with the difference in crude all-cause mortality being largest among patients $\geq 75$ years (5.6 vs. $18.2 \%, p<0.001$ ).

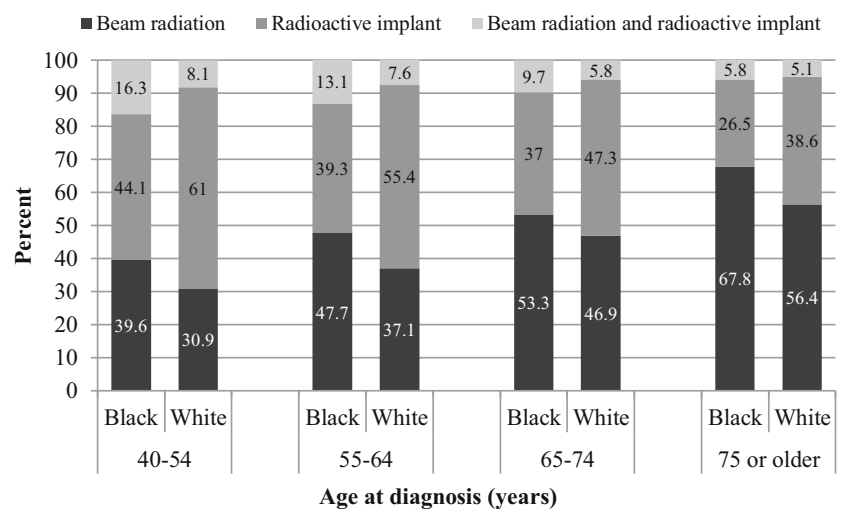

Fig. 3 Distribution of type of radiation therapy received by age group and race 
Similarly, among white patients, both crude all-cause mortality and cancer-specific mortality were lower for patients who received definitive treatment ( 2.0 vs. $5.9 \%, p<0.001$, and 0.15 vs. $0.49 \%, p<0.001$, respectively)

The distribution of patient demographic and clinical characteristics within treatment subgroups are presented in Table 2. Within each subgroup, a higher proportion of black patients were younger than 65 years, and a higher proportion of black patients had PSA levels above $4 \mathrm{ng} / \mathrm{mL}$. The crude all-cause mortality was significantly higher for black patients among patients who received radiotherapy ( $p=0.003$ ), but there was no significant difference between black and white patients on crude cancer-specific mortality for patients undergoing surgical treatment $(p=0.243)$, or radiotherapy $(p=0.717)$, and on crude all-cause mortality for patients undergoing surgical treatment $(p=0.143)$.

On multivariable logistic regression analysis, adjusting for age, SEER registry, time trend, socioeconomic factors, PSA level, and Gleason score, black patients had a $29 \%$ lower likelihood of receiving DT (adjusted odds ratio (AOR), 0.71; $95 \%$ CI, 0.67-0.76), and among patients who received DT, a $42 \%$ reduced odds of undergoing surgical treatment (AOR, $0.58 ; 95 \% \mathrm{CI}, 0.54-0.62$ ). The significant difference persisted among patients younger than 65 years (AOR for DT, 0.67; $95 \%$ CI, 0.62-0.73; AOR for surgical treatment among patients receiving DT, 0.57; $95 \% \mathrm{CI}, 0.52-0.62$ ) and among patients 65 years or older (AOR for DT, 0.88; $95 \%$ CI, $0.81-$ 0.96; AOR for surgical treatment among patients receiving DT, 0.85 ; $95 \%$ CI, 0.75-0.96). Across age groups $(<65$ and $\geq 65$ years), black patients had $45 \%$ (AOR, $1.45 ; 95 \% \mathrm{CI}$, $1.13-1.87$ ) and $56 \%$ (AOR, 1.56; $95 \%$ CI, 1.31-1.86) higher odds of all-cause mortality, respectively (Fig. 4). The age- and intervention-stratified comparisons of cancer-specific mortality are shown in Table 1. An upward trend in surgical treatment was noted for black patients while the trend in radiotherapy for black patients was downward over the study period (Fig. 5)

\section{Discussion}

The optimal management of low-risk prostate cancer continues to be debated. Our study of a relatively young cohort of African-American men with low-risk disease, using a population-based nationwide database, seeks to investigate contemporary treatment patterns for this group of patients.

With an average age of 62 years (compared to 65 years for white patients), and about $59 \%$ (compared to $49.3 \%$ for white patients) of black patients being less than 65 years, our study supports the findings of previous studies that have reported younger age for African-American men diagnosed with prostate cancer compared to other ethnic groups. Moses et al. [12]

Table 2 Distribution of patient characteristics by treatment modality and race

\begin{tabular}{|c|c|c|c|c|c|c|}
\hline \multirow[t]{2}{*}{ Characteristics } & \multicolumn{2}{|l|}{ Prostatectomy } & \multicolumn{2}{|l|}{ Radiotherapy } & \multicolumn{2}{|c|}{ No definitive treatment } \\
\hline & Black, $n=1829$ & White, $n=15,529$ & Black, $n=3437$ & White, $n=20,237$ & Black, $n=1934$ & White, $n=11,133$ \\
\hline Age (years), median (IQR) & $58(11)$ & $60(10)$ & $64(11)$ & $67(11)$ & $65(13)$ & $69(13)$ \\
\hline \multicolumn{7}{|l|}{ Age group (years), $\%$} \\
\hline $40-54$ & 33.1 & 21.7 & 13.9 & 6.0 & 12.1 & 6.7 \\
\hline $55-64$ & 46.8 & 53.2 & 39.7 & 32.5 & 36.5 & 26.6 \\
\hline $65-74$ & 19.7 & 23.8 & 38.4 & 46.6 & 37.5 & 39.2 \\
\hline 75 or older & 0.4 & 1.3 & 8.0 & 15.0 & 14.9 & 27.5 \\
\hline PSA (ng/mL), median (IQR) & $5.0(2.2)$ & $4.9(2.3)$ & $5.5(2.6)$ & $5.4(2.5)$ & $5.5(2.8)$ & $5.3(2.8)$ \\
\hline \multicolumn{7}{|l|}{ PSA (ng/mL), $\%$} \\
\hline$\leq 2.5$ & 7.9 & 10.7 & 6.8 & 7.4 & 8.7 & 11.9 \\
\hline $2.6-4$ & 16.3 & 16.5 & 9.3 & 10.5 & 9.1 & 11.7 \\
\hline $4.1-6.9$ & 59.2 & 56.3 & 57.8 & 58.0 & 54.6 & 52.1 \\
\hline $7-10$ & 16.6 & 16.6 & 26.0 & 24.1 & 27.7 & 24.3 \\
\hline \multicolumn{7}{|l|}{ Gleason Score, \% } \\
\hline $2-3$ & 0.4 & 0.3 & 0.4 & 0.3 & 0.7 & 0.7 \\
\hline $4-5$ & 3.2 & 2.5 & 2.6 & 2.4 & 4.3 & 5.2 \\
\hline 6 & 96.4 & 97.3 & 96.9 & 97.2 & 94.9 & 94.1 \\
\hline Crude all-cause mortality, $\%$ & 1.5 & 1.1 & 3.6 & 2.7 & 7.8 & 5.9 \\
\hline Crude cancer-specific mortality, $\%$ & 0.22 & 0.12 & 0.15 & 0.17 & 0.41 & 0.49 \\
\hline
\end{tabular}

Patients who received both prostatectomy and radiation therapy were not included in the table

$P S A$ prostate-specific antigen 
Fig. 4 Adjusted odds of all-cause mortality across age groups. White patients as reference

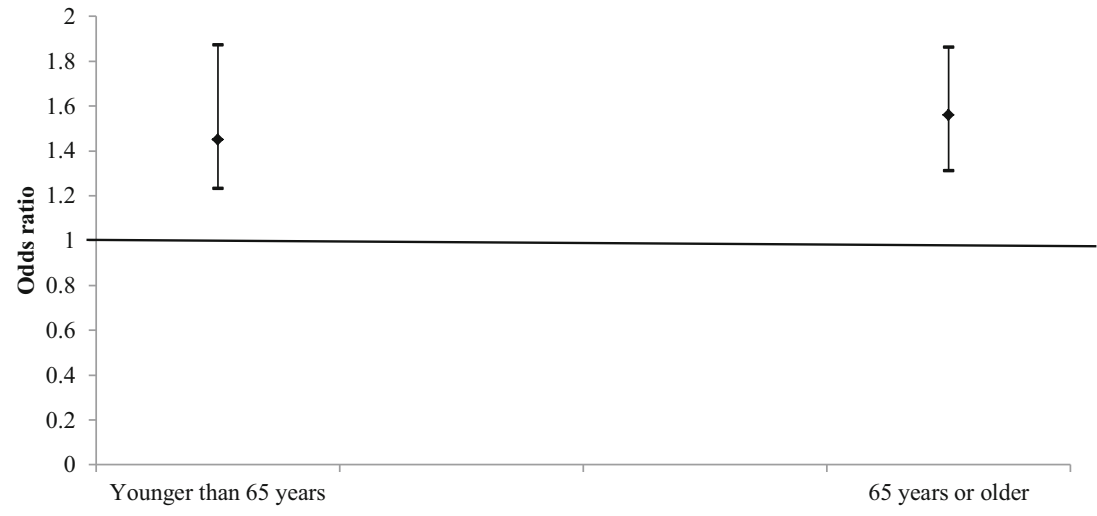

reported $77 \%$ of African-American men younger than 70 years at diagnosis of prostate cancer, compared with $68 \%$ of whites, and $72 \%$ of other ethnic groups. Also, Hoffman et al. [13] reported significantly higher proportion of African-American men compared to non-Hispanic white (53 vs $43.3 \%$ ) less than 65 years with localized prostate cancer; and a similar younger mean age of AfricanAmerican men (66.5 years) compared to other ethnic groups (68.9 and 68.1 years for White and Hispanic, respectively) was reported by Underwood et al. [14].

The rate of DT in the present study is higher than rates reported in previous population-based studies. Schymura et al. [15] reported overall DT rates for patients with clinically localized prostate cancer of $39.70 \%$ (36.74 \% for nonHispanic Blacks) and $31.42 \%$ (26.09\% for non-Hispanic Blacks) for radical prostatectomy and radiation therapy respectively; rates reported by Harlan et al. [16] for nonHispanic Blacks with clinically localized prostate cancer were 48.5 and $18.6 \%$ for radical prostatectomy and radiation therapy, respectively. Both studies included a cohort of patients who had initial diagnosis in the 1990s, thus suggesting changing treatment patterns that may have occurred over the years, and possibly, a reflection of the current trend of overtreatment of these patients.

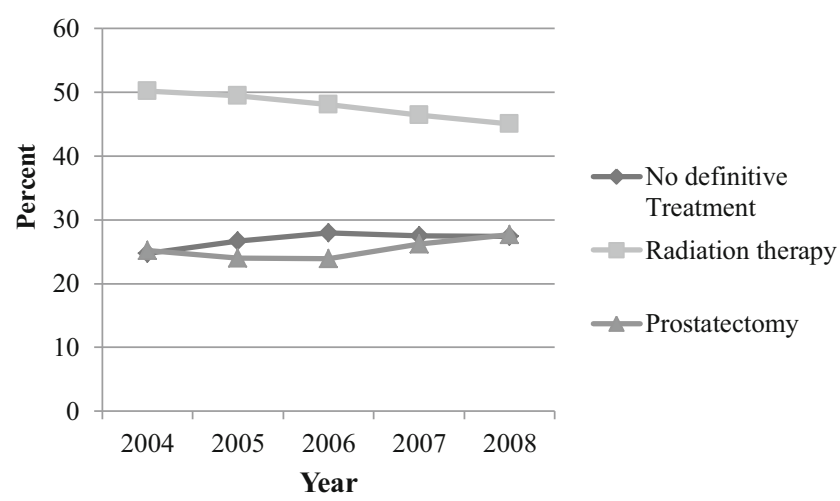

Fig. 5 Treatment trends for African-American men with low-risk prostate cancer
Our study findings of significant differences between black and white patients in the receipt of DT supports the findings of several studies that have reported racial differences in the treatment of localized prostate cancer [13-15, 17-19]. Schapira et al. [17], using a similar database as used in the present study, showed African-American men with diagnosis of localized prostate cancer to be less likely to receive aggressive treatment (radical prostatectomy or external beam radiation), and for those who received aggressive treatment, a lower likelihood of receiving radical prostatectomy. In a more recent study, Moses et al. [12], using the Cancer of the Prostate Strategic Urologic Research Endeavor (CaPSURE) database, reported that African-American men were more likely to receive androgen deprivation therapy, and less likely to receive surgery, for low-risk disease compared with their white counterparts. Similar findings have been reported $[13,15,18$, 20]. Harlan et al. [16], however, showed that racial differences in treatment did not exist among patients younger than 60 years, but was present among patients older than 60 years, with African-American men in the older age category being less likely to receive aggressive treatment. In the present study, we found that significant differences in the likelihood of receiving DT and in undergoing surgical treatment persisted even among younger (less than 65 years) and older (65 years or older) patient subgroups. Several factors have been hypothesized to influence treatment choice [21-26], with more radical prostatectomy being offered and less radiation therapy over time.

We found that older men ( 75 years or older) were less likely to receive DT, and among those who received DT, majority received radiation therapy (Fig. 2). Similar lower likelihood of receipt of DT for older patients has been reported and has been largely attributed to higher rates of comorbidity and lower life expectancy in this patient population. Although the majority of the black patients younger than 65 years in our study cohort received DT, a substantial proportion of these patients also received radiation therapy.

In the present study, both crude all-cause mortality and crude cancer-specific mortality were lower among patients 
who received DT, with the difference in crude all-cause mortality persisting even after stratifying by age group, and the gap being widest among the oldest age group. This finding may be related to the higher likelihood of not receiving DT that was noted for the older age group, thus magnifying the survival benefit of undergoing DT. Furthermore, even after controlling for potential confounding factors (including type of intervention), younger and older black patients in the present study had worse survival compared to their white counterparts. Concurrent comorbid conditions may have played a role in patient selection and subsequent outcome. In a study investigating radical prostatectomy outcome in men 65 years or older with low-risk prostate cancer, using a single institution data, Mullins et al. [27] reported excellent long-term survival in the study cohort, and this was despite the high rates of aggressive tumor found following surgery. Most of these men, however, were in excellent health, thus making a case for the use of radical prostatectomy in carefully selected group of patients. In a recent study by Liu et al. [28] on patients with low-risk prostate cancer, in which simulation models were used, the authors reported greater than expected benefit for active surveillance compared to radical prostatectomy for older patients and those with poorer baseline health status. Importantly, there is need for elderly African-American men with low-risk prostate cancer to be evaluated for possible surgical treatment, even though the tools needed for such decision-making process may be limited. Counseling these patients on the benefits of treatment should be incorporated early on in the management plan. Also, a higher level of vigilance, with follow-up re-biopsy, is needed for the subgroup of patients who would benefit more from active surveillance. The lack of information on watchful waiting or active surveillance in the SEER public-use database makes it difficult to ascertain whether or not those patients who did not receive definitive treatment in the present study received any of these other treatment options. An additional speculation about this group of patients could be loss to follow, which could be multifactorial.

One limitation of our study was the lack of information on PSA values in SEER prior to 2004, thus limiting the period covered by the study. Also, the lack of information on watchful waiting or active surveillance in SEER makes it difficult to ascertain whether or not patients who did not receive DT received any of these other treatment options. The unavailability of data on comorbidity in the SEER public-use database also meant that we could not include comorbidity in our risk-adjusted model. Despite these limitations, a major strength of our study is the large, population-based nature of the SEER database, making it generalizable.

In conclusion, our study uses a large cohort of AfricanAmerican men with early-onset low-risk prostate cancer to develop a deeper understanding of treatment and mortality trends in an ethnic group identified as having higher risk for disease progression and cause-specific mortality. The majority of these patients received DT, primarily radiotherapy. When compared to their white counterparts, black patients were significantly less likely to receive DT, of those receiving DT, less likely to undergo surgical treatment, and regardless of age group, had worse survival. As expected, older men were less likely to receive definitive treatment. However, a surprising observation was the striking survival difference among elderly men between those who received definitive treatment and those who did not receive definitive treatment. Further investigation of these differences may provide insight for better risk stratification.

Conflict of Interest All authors declare that they have no conflict of interest.

Informed Consent No animal or human studies were carried out by the authors for this article.

\section{References}

1. Siegel R, Naishadham D, Jemal A. Cancer statistics, 2012. CA Cancer J Clin. 2012;62:10-29.

2. Mettlin CJ, Murphy GP, McGinnis LS, Menck HR. The National Cancer Data Base report on prostate cancer. American College of Surgeons Commission on Cancer and the American Cancer Society. Cancer. 1995;76:1104-12.

3. Mettlin CJ, Murphy GP, Cunningham MP, Menck HR. The National Cancer Data Base report on race, age, and region variations in prostate cancer treatment. Cancer. 1997;80:1261-6.

4. Hoffman RM, Gilliland FD, Eley JW, et al. Racial and ethnic differences in advanced-stage prostate cancer: the Prostate Cancer Outcomes Study. J Natl Cancer Inst. 2001;93:388-95.

5. Freedland SJ, Isaacs WB. Explaining racial differences in prostate cancer in the United States: sociology or biology. Prostate. 2005;62: 243-52.

6. Cooperberg MR, Broering JM, Kantof PW, Carroll PR. Contemporary trends in low risk prostate cancer: risk assessment and treatment. J Urol. 2007;178:S14-9.

7. Kollmeier MA, Stock RG, Cesaretti J, Stone NN. Urinary morbidity and incontinence following transurethral resection of the prostate after brachytherapy. J Urol. 2005;173(3):808-12.

8. Frey CM, McMillen MM, Cowan CD, Horm JW, Kessler LG. Representativeness of the surveillance, epidemiology, and end results program data: recent trends in cancer mortality rates. J Natl Cancer Inst. 1992;84(11):872-7.

9. Surveillance, Epidemiology, and End Results (SEER) Program (www.seer.cancer.gov) Research Data (1973-2008) National Cancer Institute, DCCPS, Surveillance Research Program, Surveillance Systems Branch, released May 2011.

10. Shao YH, Demissie K, Shih W, Mehta AR, Stein MN, Roberts CB, et al. Contemporary risk profile of prostate cancer in the United States. J Natl Cancer Inst. 2009;101(18):1280-3.

11. D'Amico AV, Whittington R, Malkowicz SB, Cote K, Loffredo M, Schultz D, et al. Biochemical outcome after radical prostatectomy or external beam radiation therapy for patients with clinically localized prostate carcinoma in the prostate specific antigen era. Cancer. 2002;95(2):281-6. 
12. Moses K, Paciorek A, Penson D, Carroll P, Master V. Impact of ethnicity on primary treatment choice and mortality in men with prostate cancer: data from CaPSURE. J Clin Oncol. 2010;28(6): 1069-74.

13. Hoffman RM, Harlan LC, Klabunde CN, Gilliland FD, Stephenson RA, Hunt WC, et al. Racial differences in initial treatment for clinically localized prostate cancer. J Gen Intern Med. 2003;18(10): 845-53.

14. Underwood III W, Jackson J, Wei JT, Dunn R, Baker E, DeMonner S, et al. Racial treatment trends in localized/regional prostate carcinoma: 1992-1999. Cancer. 2005;103(3):538-45.

15. Schymura MJ, Kahn AR, German RR, Hsieh M, Cress RD, Finch JL, et al. Factors associated with initial treatment and survival for clinically localized prostate cancer: results from the CDC-NPCR Patters of Care Study (PoC1). BMC Cancer. 2010;10:152.

16. Harlan LC, Potosky A, Gilliland FD, Hoffman R, Albertsen PC, Hamilton AS, et al. Factors associated with initial therapy for clinically localized prostate cancer: prostate cancer outcomes study. J Natl Cancer Inst. 2001;93(24):1864-71.

17. Schapira MM, McAuliffe TL, Nattinger AB. Treatment of localized prostate cancer in African-American compared with Caucasian men. Less use of aggressive therapy or comparable disease. Med Care. 1995;33(11):1079-88.

18. Harlan L, Brawley O, Pommerenke F, Wali P, Kramer B. Geographic, age, and racial variation in the treatment of local/regional carcinoma of the prostate. J Clin Oncol. 1995;13(1):93-100.

19. Richert-Boe KE, Weinmann S, Shapiro JA, Rybicki BA, Enger SM, Van Den Eeden SK, et al. Racial differences in treatment of earlystage prostate cancer. Urology. 2008;71(6):1172-6.
20. Morris CR, Snipes KP, Schlag R, Wright WE. Sociodemographic factors associated with prostatectomy utilization and concordance with the physician data query for prostate cancer (United States). Cancer Causes Control. 1999;10(6):503-11.

21. Steginga SK, Occhipinti S, Gardiner RA, Yaxley J, Heathcote P. Making decisions about treatment for localized prostate cancer. BJU Int. 2002;89(3):255-60.

22. Wagner SE, Drake BF, Elder D, Herbert JR. Social and clinical predictors of prostate cancer treatment decisions among men in South Carolina. Cancer Causes Control. 2011;22(11):1597-606.

23. Zeliadt SB, Ramsey SD, Penson DF, Hall IJ, Ekwueme DU, Stroud $\mathrm{L}$, et al. Why do men choose one treatment over another? a review of patient decision making for localized prostate cancer. Cancer. 2006;106(9):1865-74.

24. Chapple A, Ziebland S, Herxheimer A, McPherson A, Shepperd S, Miller R. Is 'watchful waiting' a real choice for men with prostate cancer? A qualitative study. BJU Int. 2002;90(3):257-64.

25. Shankar S, Selvin E, Alberg AJ. Perceptions of cancer in an AfricanAmerican community: a focus group report. Ethn Dis. 2002;12(2): 276-83.

26. Davison BJ, Oliffe JL, Pickles T, Mroz L. Factors influencing men undertaking active surveillance for the management of low-risk prostate cancer. Oncol Nurs Forum. 2009;36(1):89-96.

27. Mullins JK, Han M, Pierorazio PM, Partin AW, Carter HB. Radial prostatectomy outcome in men 65 years old or older with low risk prostate cancer. J Urol. 2012;187(5):1620-5.

28. Liu D, Lehmann HP, Frick KD, Carter HB. Active surveillance versus surgery for low risk prostate cancer: a clinical decision analysis. J Urol. 2012;187(4):1241-6. 